\title{
Effects of Obstructive Sleep Apnea Hypopnea Syndrome on Blood Pressure and C-Reactive Protein in Male Hypertension Patients
}

\author{
Fan Li ${ }^{\mathrm{a}}$, Hui Huang ${ }^{\mathrm{a}}$, Ligong Song ${ }^{\mathrm{a}}$, Hua Hao ${ }^{\mathrm{a}}$, Mingzhong Yinga, b
}

\begin{abstract}
Background: The influences of obstructive sleep apnea hypopnea syndrome (OSAHS) on blood pressure and C-reactive protein (CRP) were observed, and the underlying mechanism was investigated.

Methods: Respiratory sleep monitoring was performed on 188 male patients who were newly diagnosed with hypertension. Based on the apnea hypopnea index (AHI) results, patients were divided into a normal control group $(\mathrm{AHI} \leq 5, \mathrm{n}=35)$, a mild OSAHS group $(5<\mathrm{AHI}$ $\leq 15, \mathrm{n}=28)$, a moderate OSAHS group $(15<\mathrm{AHI} \leq 30, \mathrm{n}=57)$, and a severe OSAHS group (AHI $>30, \mathrm{n}=68)$. Ambulatory blood pressure monitoring was conducted on patients in each group, and blood samples were collected to detect indicators, including fasting blood glucose (FBG), triglyceride (TG), low-density lipoprotein-cholesterol (LDL-C), high-density lipoprotein-cholesterol (HDL-C), and highsensitivity CRP (hs-CRP).
\end{abstract}

Results: TG and hs-CRP in patients in the moderate and severe OSAHS groups were higher than those in the normal control group ( $\mathrm{P}$ $<0.01, \mathrm{P}<0.05)$. Additionally, their mean nocturnal systolic blood pressure (nSBP) and nocturnal diastolic blood pressure (nDBP) were higher than those in the normal control group $(\mathrm{P}<0.01, \mathrm{P}<0.05)$. However, the percentage of blood pressure reduction at night was significantly lower than that in the normal control group $(\mathrm{P}<0.01)$. AHI and hs-CRP positively correlated with $\mathrm{nSBP}$ (adjusted $\left.\mathrm{R}^{2}=0.46\right)$ and $\mathrm{nDBP}$ (adjusted $\mathrm{R}^{2}=0.38$ ) and negatively correlated with the nocturnal blood pressure reduction percentage (adjusted $\mathrm{R}^{2}=0.48$ ).

Conclusion: Moderate and severe OSAHS induced increases in nocturnal blood pressure and CRP content in the body, resulting in further damage to the circadian rhythms of blood pressure.

Keywords: Obstructive sleep apnea hypopnea syndrome; Hypertension; C-reactive protein

Manuscript accepted for publication November 17, 2015

aThe International Medical Center of the People's Liberation Army General Hospital, District 3, Division 2, 28 Fuxin Road, Beijing 100853, China

${ }^{b}$ Corresponding Author: Mingzhong Ying, The International Medical Center of the People's Liberation Army General Hospital, District 3, Division 2, 28 Fuxin Road, Beijing 100853, China. Email: lifan76@sohu.com

doi: http://dx.doi.org/10.14740/jocmr2409w

\section{Introduction}

Hypertension is one of the most important risk factors for patients with cardiovascular and cerebrovascular diseases. Achieving blood pressure control within the standard range has important significance for the reduction of cardiovascular mortality. However, studies have shown that when hypertension patients also had obstructive sleep apnea hypopnea syndrome (OSAHS), their blood pressure fluctuations were more obvious than those in general hypertension patients, and they had difficultly achieving control within the standard range [1]. Currently, it is known that hypoxemia caused by OSAHS influences blood pressure. However, are other factors involved in this regulation? As an inflammatory substance, C-reactive protein (CRP) is considered an important pathophysiological factor in the development and progression of hypertension. Therefore, this study performed respiratory sleep monitoring, ambulatory blood pressure monitoring, and laboratory tests on hypertension patients to investigate the correlations between OSAHS, blood pressure fluctuations, and CRP to guide clinical treatment.

\section{Patients and Methods}

\section{Clinical data}

A total of 188 male hypertension patients diagnosed and treated in our department from June 2014 to May 2015 who all conformed to the diagnostic criteria of the "2014 evidence-based guideline for the management of high blood pressure in adults" (JNC8) [2] were enrolled in this study. They had systolic blood pressure (SBP) $\geq 140 \mathrm{~mm} \mathrm{Hg}$ and/or diastolic blood pressure (DBP) $\geq 90 \mathrm{~mm} \mathrm{Hg}$, they had clearly been diagnosed with hypertension, and they had not started anti-hypertensive drug treatment. Patients with mental disorders, significant upper respiratory tract obstructions, upper respiratory tract infections, diabetes mellitus, histories of cerebral hemorrhage or infarction, cardiac insufficiency (higher than grade III in the New York Heart Association (NYHA) Functional Classification), hypothyroidism, late-stage malignant tumors, and severe hepatic and renal dysfunction were excluded. The ages of the patients were between 38 and 62 years (mean $46 \pm 7$ years). The study proto- 
Table 1. Comparison of Clinical and Biochemical Data Among All Groups

\begin{tabular}{lllll}
\hline Item & $\begin{array}{l}\text { Normal control } \\
\text { group }(\mathbf{n}=\mathbf{3 5})\end{array}$ & $\begin{array}{l}\text { Mild OSAHS } \\
\text { group }(\mathbf{n}=\mathbf{2 8})\end{array}$ & $\begin{array}{l}\text { Moderate OSAHS } \\
\text { group }(\mathbf{n}=\mathbf{5 7})\end{array}$ & $\begin{array}{l}\text { Severe OSAHS } \\
\text { group }(\mathbf{n}=\mathbf{6 8})\end{array}$ \\
\hline Age $(\mathrm{year})$ & $43 \pm 9$ & $41 \pm 12$ & $45 \pm 8$ & $45 \pm 7$ \\
BMI $\left(\mathrm{kg} / \mathrm{m}^{2}\right)$ & $25.62 \pm 2.79$ & $26.07 \pm 4.11$ & $26.80 \pm 2.54^{*}$ & $27.15 \pm 2.20^{*}$ \\
\hline FBG $(\mathrm{mmol} / \mathrm{L})$ & $5.07 \pm 1.61$ & $5.29 \pm 1.78$ & $5.54 \pm 1.43$ & $5.47 \pm 1.26$ \\
TG $(\mathrm{mmol} / \mathrm{L})$ & $1.82 \pm 1.80$ & $2.74 \pm 2.02$ & $2.98 \pm 1.97 \dagger$ & $2.94 \pm 1.79 \dagger$ \\
LDL-C $(\mathrm{mmol} / \mathrm{L})$ & $2.88 \pm 1.35$ & $3.15 \pm 1.69$ & $3.07 \pm 1.41$ & $3.21 \pm 1.32$ \\
HDL-C $(\mathrm{mmol} / \mathrm{L})$ & $1.14 \pm 0.47$ & $1.06 \pm 0.81$ & $0.98 \pm 0.35$ & $0.92 \pm 0.29$ \\
hs-CRP $(\mathrm{mg} / \mathrm{L})$ & $2.33 \pm 2.82$ & $3.68 \pm 6.56$ & $5.71 \pm 8.17^{*}$ & $6.45 \pm 7.25 \dagger$ \\
\hline
\end{tabular}

Compared with the normal control group, ${ }^{*} \mathrm{P}<0.05$ and $\dagger \mathrm{P}<0.01$.

col was approved by the Ethics Committee of the PLA General Hospital and all the patients provided written informed consent, according to the Declaration of Helsinki, prior to inclusion.

\section{Data collection}

The patients' ages, heights, and body weights were recorded. The body mass index (BMI) was calculated for each patient according to the formula: BMI = body weight $(\mathrm{kg}) /$ height $^{2}\left(\mathrm{~m}^{2}\right)$.

\section{Sleep monitoring and grouping}

All study subjects received examinations using a portable sleep apnea recording device (ApneaLink ${ }^{\mathrm{TM}}$, ResMed, Australia). On the first day of admission, the recording time was longer than $7 \mathrm{~h}$. On the examination day, drinking alcohol and coffee and taking sedatives and sleeping pills were prohibited to avoid interfering with the assessments. The monitoring items included posture, nasal airflow, thoracoabdominal movement, and the minimum blood oxygen saturation $\left(\mathrm{SpO}_{2}\right)$. On the next day, the sleep monitoring results of all patients were analyzed according to the 2003 Chinese OSAHS treatment guidelines (draft), of which the apnea hypopnea index $(\mathrm{AHI})=(($ number of apnea events + number of hypopnea events)/sleep time $(\min )) \times 60$. Based on the detected AHI values, the 188 patients were divided into 35 cases in the normal control group $(\mathrm{AHI} \leq 5), 28$ cases in the mild OSAHS group $(5<\mathrm{AHI} \leq 15)$, 57 cases in the moderate OSAHS group $(15<\mathrm{AHI} \leq 30)$, and 68 cases in the severe OSAHS group (AHI > 30).

\section{Ambulatory blood pressure monitoring}

Starting from 8:00 - 9:00, 24-h ambulatory blood pressure monitoring using a portable type 90217 non-invasive cuff automatic blood pressure monitor (SPACELABS ${ }^{\mathrm{TM}}$, USA) was conducted on all subjects. During the monitoring period, patients resumed their normal daily lives. The effective recording time of blood pressure monitoring had to be longer than $20 \mathrm{~h}$. The effective reading of blood pressure $>85 \%$ of the time was regarded as qualified; otherwise, the readings had to be taken again. Com- puters automatically performed statistical analyses of ambulatory blood pressure monitoring data. The observation parameters included mean daytime systolic blood pressure (dSBP), mean daytime diastolic blood pressure (dDBP), mean nocturnal SBP (nSBP), and mean nocturnal DBP (nDBP). In addition, the nocturnal blood pressure reduction percentage was calculated using the formula $(\mathrm{dSBP}-\mathrm{nSBP}) / \mathrm{dSBP} \times 100 \%$, which represented the amplitudes of circadian blood pressure changes in patients.

\section{Laboratory examination}

During the next morning, when receiving respiratory sleep monitoring (after fasting overnight for $12 \mathrm{~h}$ ), patients' cubital vein blood samples were collected to detect fasting blood glucose (FBG), triglyceride (TG), high-density lipoprotein-cholesterol (HDL-C), low-density lipoprotein-cholesterol (LDLC), and high-sensitivity CRP (hs-CRP). FBG, TG, HDL-C and LDL-C were measured using the 7200 automatic biochemical analyzer (Japan), and hs-CRP was measured using an enzymelinked immunosorbent assay (ELISA); the reagent kit was purchased from Shanghai Senxiong BioTechnology Co., Ltd. The detection procedure and calculation were performed strictly according to the instructional manual.

\section{Statistical methods}

Statistical analysis was performed using the software SPSS version 17.0. Measurement data were presented as $\bar{\chi} \pm \mathrm{s}$. Comparison of mean values between groups was performed using the $\mathrm{F}$ test. Factors that affected blood pressure were obtained using a stepwise multiple linear regression analysis. $\mathrm{P}<0.05$ indicated that the difference was statistically significant.

\section{Results}

\section{Comparison of clinical and biochemical data}

According to the respiratory sleep monitoring results, the numbers of cases in each group were 35 cases in the normal control 
Table 2. Comparison of Mean Nocturnal Blood Pressure in Each Group

\begin{tabular}{|c|c|c|c|c|c|c|}
\hline Group & Case number & dSBP (mm Hg) & dDBP (mm Hg) & nSBP (mm Hg) & nDBP (mm Hg) & $\begin{array}{l}\text { Nocturnal blood } \\
\text { pressure reduction } \\
\text { percentage }(\%)\end{array}$ \\
\hline Normal control group & 35 & $137.3 \pm 14.6$ & $88.1 \pm 10.4$ & $117.9 \pm 11.2$ & $77.4 \pm 8.5$ & $13.5 \pm 5.4$ \\
\hline Mild OSAHS group & 28 & $135.4 \pm 18.2$ & $89.2 \pm 12.1$ & $119.1 \pm 12.3$ & $82.0 \pm 11.4$ & $11.8 \pm 8.6$ \\
\hline Moderate OSAH group & 57 & $136.8 \pm 13.9$ & $90.4 \pm 9.7$ & $128.6 \pm 17.1 *$ & $86.7 \pm 19.3^{*}, \dagger$ & $5.8 \pm 4.2 *$ \\
\hline Severe OSAHS group & 68 & $138.6 \pm 11.5$ & $91.7 \pm 11.2$ & $134.3 \pm 16.4^{*},+$ & $90.3 \pm 17.1 *,+$ & $2.9 \pm 2.1 *,+$ \\
\hline
\end{tabular}

Compared with the normal control group, ${ }^{*} P<0.01$; compared with the mild OSAHS group, $† P<0.05$ and $\ddagger P<0.01$.

group, 28 cases in the mild OSAHS group, 57 cases in the moderate OSAHS group, and 68 cases in the severe OSAHS group. Compared to the normal control group, patients in the OSAHS groups had increased BMIs and TG and hs-CRP levels; of these measurements, the changes in patients in the moderate and severe groups were significantly different $(\mathrm{P}<0.01, \mathrm{P}<0.05)$. Other indicators did not have changes $(\mathrm{P}>0.05)$ (Table 1).

\section{Blood pressure changes}

The dSBP and dDBP values of hypertension patients among the different groups were not significantly different $(\mathrm{P}>0.05)$. However, the $\mathrm{nSBP}$ and $\mathrm{nDBP}$ values in patients in the three OSAHS groups were all higher than those in the normal control group. The nocturnal blood pressure reduction percentage significantly decreased; when the degree of OSAHS was more serious, the percentage of the reduction of nocturnal blood pressure was lower, and the difference between the moderate and severe OSAHS groups and the normal control group was statistically significant $(\mathrm{P}<0.01)$. In addition, compared to the mild OSAHS group, the nSBP and nDBP values in patients in the moderate and severe OSAHS groups also significantly increased $(\mathrm{P}<0.01, \mathrm{P}<0.05)$, and the nocturnal blood pressure reduction percentage significantly decreased $(\mathrm{P}<0.01)$, while other indicators did not change $(\mathrm{P}>0.05)$ (Table 2$)$.

\section{Correlation analysis}

The above examination results showed that the BMIs and the TG and hs-CRP levels of hypertension patients combined with
OSAHS were all higher than those in general hypertension patients. In addition, the nocturnal blood pressure of hypertension patients combined with OSAHS was higher than that in general hypertension patients. Therefore, nSBP, nDBP, and the nocturnal blood pressure reduction percentage were used as dependent variables, and AHI, BMI, TG, and hs-CRP were used as independent variables to perform the stepwise multiple regression analysis to identify factors that affected nocturnal blood pressure. AHI and hs-CRP showed independent positive correlations with nSBP (adjusted $\mathrm{R}^{2}=0.46$ ) and $\mathrm{nDBP}$ (adjusted $\mathrm{R}^{2}=0.38$ ) and an independent negative correlation with the nocturnal blood pressure reduction percentage (adjusted $\mathrm{R}^{2}$ $=0.48)($ Table 3$)$.

\section{Discussion}

OSAHS is an airway obstruction and sleep apnea phenomenon with several causative factors. The main manifestations are daytime sleepiness and recurrent airway obstruction during sleep at night. OSAHS mainly occurs in obese and older people. More and more studies have discovered and confirmed that OSAHS is an independent risk factor for hypertension and is closely associated with hypertension [3]. Results in this study indicated that the influence of OSAHS on blood pressure mainly occurred at night. The $\mathrm{nSBP}$ and $\mathrm{nDBP}$ values of hypertension patients combined with OSAHS both increased to a certain extent. This influence was even more evident in patients with moderate to severe OSAHS. More and more evidence indicated that when the mean blood pressure level was the same or similar, hypertension patients with decreased or nonexistent circadian rhythms of blood pressure had sig-

Table 3. Analysis of Correlation Factors That Influenced Nocturnal Blood Pressure Changes in Patients

\begin{tabular}{llllll}
\hline Dependent variable & Independent variable & Adjusted $\mathbf{R}^{2}$ & $\mathbf{P}^{*}$ & B & $\mathbf{P}_{\dagger}$ \\
\hline nSBP & AHI & 0.34 & $<0.01$ & 1.72 & $<0.01$ \\
& hs-CPR & 0.12 & $<0.01$ & 0.88 & $<0.01$ \\
nDBP & AHI & 0.29 & $<0.01$ & 1.64 & $<0.01$ \\
& hs-CRP & 0.09 & $<0.01$ & 0.83 & $<0.01$ \\
Nocturnal blood pressure reduction percentage & AHI & 0.30 & $<0.01$ & -0.11 & $<0.01$ \\
& hs-CRP & 0.18 & $<0.01$ & -0.35 & $<0.01$ \\
\hline
\end{tabular}

${ }^{*} \mathrm{P}$ value of the adjusted $\mathrm{R}^{2} ; \dagger P$ value of $B$ 
nificantly increased risks for the development of target organ injury and cardiovascular and cerebrovascular events; in addition, there is a vicious cycle between target organ injury and abnormal circadian rhythms of blood pressure [4]. Normal blood pressure displays a low in the nighttime and high in the daytime rhythm; in fact, the nighttime blood pressure is lower than that at daytime by at least $10 \%$. During changes in the circadian rhythms of blood pressure, the mean blood pressure at night might be even higher than that at daytime. In this study, we found that hypertension patients with moderate to severe OSAHS had significant decreases of the nocturnal blood pressure reduction percentage; in fact, this measurement was an important indicator reflecting the circadian rhythm of blood pressure. Study results have shown that the nocturnal blood pressure reduction percentage is clearly correlated with OSAHS. Therefore, we considered that moderate and severe OSAHS would influence the circadian rhythm of blood pressure; in addition, the incidence of clinical endpoint events among hypertension patients with OSAHS was higher than that of general hypertension patients.

The main pathological feature of OSAHS is intermittent hypoxia. During sleep, the recurrent hypoxemia and hypercapnia in OSAHS patients can stimulate the central nervous system and the peripheral chemoreceptors, which already have increased sensitivity, to increase the activity of the sympathetic nervous system, thus increasing blood pressure. In addition, repeated awakening can further stimulate sympathetic nerve excitation to increase plasma levels of catecholamine, renin, and angiotensin II, thus causing peripheral blood vessel constriction and eventually causing an increase in nocturnal blood pressure.

In addition to its influence on the sympathetic nervous system, hypoxia can also induce oxidative imbalances in the body. In recent years, scholars proposed the concept that OSAHS was an oxidative stress disorder [6], which meant that it was a pathophysiological process of hypoxia followed by the activation of oxidative stress. Hypoxemia and hypercapnia can produce large amounts of oxygen free radicals in the body, eventually changing the oxidative stress status in the body and inducing pathological inflammatory responses; therefore, the important inflammatory factor component, CRP, is also affected. CRP is mainly synthesized in and secreted by the liver. It is connected by five polypeptide subunits with the same glycosylated structure. Because its levels are significantly increased in hypertension patients, it is currently regarded as an independent risk factor for hypertension [7]. Studies have shown that CRP can aggravate hypertension via several mechanisms. 1) CRP participates in systemic and local inflammatory responses to damage vascular endothelial cells, thus reducing the release of nitric oxide and prostaglandins; additionally, nitric oxide is a powerful vasodilation factor. 2) CRP can promote the thickening of vascular intima to promote the formation and progression of atherosclerosis, thus causing vascular remodeling, increasing resistance, and reducing vascular reactivity to endothelium-dependent vasodilators, eventually resulting in decreases in the blood flow speed during the diastolic period and the mean blood flow speed to aggravate vascular sclerosis and elevate blood pressure [8,9]. Previous studies have already confirmed that CRP is associated with OSAHS [10]. Our studies also obtained similar results. We suggest that second- ary oxidative stress responses resulting from OSAHS-induced intermittent hypoxia constitute an important reason for the elevated serum CRP levels in OSAHS patients and that elevated CRP will further influence blood pressure to cause an abnormal circadian rhythm of blood pressure.

\section{Conclusions}

In summary, intermittent hypoxia induced by OSAHS increases blood pressure through its influences on the sympathetic nervous system. In addition, OSAHS also influences the oxidative stress balance and increases inflammatory factor CRP levels in the body to further change the circadian rhythm of blood pressure. Therefore, an effective improvement of OSAHS has important significance in the control of blood pressure within the standard range.

\section{References}

1. Somers VK, White DP, Amin R, Abraham WT, Costa F, Culebras A, Daniels S, et al. Sleep apnea and cardiovascular disease: an American Heart Association/American College of Cardiology Foundation Scientific Statement from the American Heart Association Council for High Blood Pressure Research Professional Education Committee, Council on Clinical Cardiology, Stroke Council, and Council on Cardiovascular Nursing. J Am Coll Cardiol. 2008;52(8):686-717.

2. James PA, Oparil S, Carter BL, Cushman WC, Dennison-Himmelfarb C, Handler J, Lackland DT, et al. 2014 evidence-based guideline for the management of high blood pressure in adults: report from the panel members appointed to the Eighth Joint National Committee (JNC 8). JAMA. 2014;311(5):507-520.

3. Reishtein JL. Obstructive sleep apnea: a risk factor for cardiovascular disease. J Cardiovasc Nurs. 2011;26(2):106116.

4. Bastos JM, Bertoquini S, Silva JA, Polonia J. Relationship between ambulatory blood pressure monitoring values and future occurrence of ischemic cerebrovascular and coronary events in hypertensive patients. Rev Port Cardiol. 2006;25(3):305-316.

5. Liu DS, Ye JF, Zhong QY. Clinical observation of combined treatment in patients with hypertension complicated with obstructive sleep apnea hypopnea syndrome. Lingnan J Emerg Med. 2008;13(4):245-246.

6. Kent BD, Ryan S, McNicholas WT. Obstructive sleep apnea and inflammation: relationship to cardiovascular comorbidity. Respir Physiol Neurobiol. 2011;178(3):475481.

7. Bautista LE, Lopez-Jaramillo P, Vera LM, Casas JP, Otero AP, Guaracao AI. Is C-reactive protein an independent risk factor for essential hypertension? J Hypertens. 2001;19(5):857-861.

8. Perticone F, Maio R, Sciacqua A, Andreozzi F, Iemma $\mathrm{G}$, Perticone $\mathrm{M}$, et al. Endothelial dysfunction and $\mathrm{C}$ reactive protein are risk factors for diabetes in essential 
hypertension. Diabetes. 2008;57(1):167-171

9. Dotsenko O, Chaturvedi N, Thom SA, Wright AR, Mayet J, Shore A, Schalkwijk C, et al. Platelet and leukocyte activation, atherosclerosis and inflammation in European and South Asian men. J Thromb Haemost. 2007;5(10):2036-
2042.

10. Guven SF, Turkkani MH, Ciftci B, Ciftci TU, Erdogan Y. The relationship between high-sensitivity C-reactive protein levels and the severity of obstructive sleep apnea. Sleep Breath. 2012;16(1):217-221. 